\title{
Risk factors for medication non-adherence among atrial fibrillation patients
}

Stephanie R. Reading ${ }^{1}$, Mary Helen Black${ }^{1}$, Daniel E. Singer ${ }^{2}$, Alan S. Go ${ }^{3,4}$, Margaret C. Fang ${ }^{5}$, Natalia Udaltsova ${ }^{3}$, Teresa N. Harrison ${ }^{1}$, Rong X. Wei', In-Lu Amy Liu' ${ }^{1}$, Kristi Reynolds ${ }^{1 *}$ (i) and for the ATRIA-CVRN Investigators

\begin{abstract}
Background: Atrial fibrillation (AF) patients are routinely prescribed medications to prevent and treat complications, including those from common co-occurring comorbidities. However, adherence to such medications may be suboptimal. Therefore, we sought to identify risk factors for general medication non-adherence in a population of patients with atrial fibrillation.

Methods: Data were collected from a large, ethnically-diverse cohort of Kaiser Permanente Northern and Southern California adult members with incident diagnosed AF between January 1, 2006 and June 30, 2009. Self-reported questionnaires were completed between May 1, 2010 and September 30, 2010, assessing patient socio-demographics, health behaviors, health status, medical history and medication adherence. Medication adherence was assessed using a previously validated 3-item questionnaire. Medication non-adherence was defined as either taking medication(s) as the doctor prescribed $75 \%$ of the time or less, or forgetting or choosing to skip one or more medication(s) once per week or more. Electronic health records were used to obtain additional data on medical history. Multivariable logistic regression analyses examined the associations between patient characteristics and self-reported general medication adherence among patients with complete questionnaire data.
\end{abstract}

Results: Among 12,159 patients with complete questionnaire data, 6.3\% $(n=771)$ reported medication non-adherence. Minority race/ethnicity versus non-Hispanic white, not married/with partner versus married/with partner, physical inactivity versus physically active, alcohol use versus no alcohol use, any days of self-reported poor physical health, mental health and/or sleep quality in the past 30 days versus 0 days, memory decline versus no memory decline, inadequate versus adequate health literacy, low-dose aspirin use versus no low-dose aspirin use, and diabetes mellitus were associated with higher adjusted odds of non-adherence, whereas, ages $65-84$ years versus $<65$ years of age, a Charlson Comorbidity Index score $\geq 3$ versus 0 , and hypertension were associated with lower adjusted odds of non-adherence.

Conclusions: Several potentially preventable and/or modifiable risk factors related to medication non-adherence and a few non-modifiable risk factors were identified. These risk factors should be considered when assessing medication adherence among patients diagnosed with AF.

Keywords: Adherence, Epidemiology, Anticoagulants, Warfarin, Cardiology

\footnotetext{
* Correspondence: kristi.reynolds@kp.org

Stephanie R. Reading: Work was completed as a post-doctoral research

fellow. Recently transitioned to the Center for Observational Research,

Amgen Inc, Thousand Oaks, CA

'Department of Research and Evaluation, Kaiser Permanente Southern

California, 100 S. Los Robles Ave., 2nd floor, Pasadena, CA 91101, USA

Full list of author information is available at the end of the article
}

(c) The Author(s). 2019 Open Access This article is distributed under the terms of the Creative Commons Attribution 4.0 International License (http://creativecommons.org/licenses/by/4.0/), which permits unrestricted use, distribution, and reproduction in any medium, provided you give appropriate credit to the original author(s) and the source, provide a link to the Creative Commons license, and indicate if changes were made. The Creative Commons Public Domain Dedication waiver (http://creativecommons.org/publicdomain/zero/1.0/) applies to the data made available in this article, unless otherwise stated. 


\section{Background}

Atrial fibrillation (AF) is the most common clinicallysignificant adult arrhythmia [1, 2]. In 2010, 5.2 million people in the United States were estimated to have AF and a projected 12.1 million people were expected to have AF by the year 2030 [3]. For individuals with AF, the most frequent cardiovascular complications include ischemic stroke, heart failure and sudden cardiac death [4]. To prevent such complications, medications, including anticoagulants, are prescribed to lower the risk of ischemic stroke and other arterial thromboembolisms [5]. Medications for heart rate control and/or medications for rhythm control are also routinely prescribed [6-8]. Nevertheless, adherence to such prescribed medications is suboptimal and can translate into an increased risk of treatment failure, hospitalizations and early mortality [9-15].

In addition to the potential occurrence of AF-specific complications, many patients with AF present with non-cardiovascular specific comorbidities [16-19]. One study reported that $98 \%$ of patients with AF had at least one comorbidity [16]. The most commonly reported non-cardiovascular comorbidity was urologic disorders (62\%) followed by chronic pain (61\%), respiratory (42\%), gastrointestinal (41\%), sleep (29\%), psychiatric (28\%), cancer $(26 \%)$ and dermatologic $(26 \%)$ conditions. However, little is known about medication adherence to the treatment of these comorbidities and limited data exist regarding predictors of AF-specific medication non-adherence $[15,20]$. It may be that patients with $\mathrm{AF}$ present as a unique sub-population among those with cardiovascular disease. According to the American Heart Association, many people with AF don't recognize the seriousness of their illness (>65\%) [21] and may be less adherent to their medication compared to those in the general cardiovascular disease population. Additionally, patients with AF may not experience symptoms [21], leading to potentially lower adherence to medication. Although these instances are specific to treatments for AF, these behaviors may carry over into adherence to their other medication regimens.

It has been suggested, in studies assessing AF-specific medication adherence, that younger age may be one possible risk factor for non-adherence [22], but the findings for gender [23, 24], socioeconomic status $[22,25]$ and comorbidities $[26,27]$ are mixed. Additionally, dementia, mental function and the complexity of the dosing regimen have been found to decrease medication adherence in patients with $\mathrm{AF}[20,22,28]$. Identifying those $\mathrm{AF}$ patients at greatest risk for general medication non-adherence remains a difficult task. To address this knowledge gap, we evaluated the associations between select patient characteristics and self-reported medication adherence within a large, ethnically diverse population of adults with incident diagnosed AF to elucidate those patients who may be at greatest risk for medication non-adherence.

\section{Methods \\ Setting}

Kaiser Permanente Northern California (KPNC) and Kaiser Permanente Southern California (KPSC) are two integrated healthcare delivery systems that currently provide comprehensive care to over 8 million individuals throughout the state of California. These $>8$ million individuals represent a socio-demographically diverse population that is highly characteristic of the statewide population [29-31]. Complete details of the healthcare services that these individuals receive are captured through structured administrative and clinical databases managed through the EpicCare system (Epic Systems, Verona, WI).

\section{Study population}

The present investigation included KPNC and KPSC members who were part of the Anticoagulation and Risk Factors in Atrial Fibrillation - Cardiovascular Research Network (ATRIA-CVRN) cohort [32]. Details of this cohort have been previously described [1,32-35]. In short, patients 21 years of age or older who were diagnosed with incident AF or atrial flutter between January 1, 2006 and June 30, 2009 were included (Fig. 1). Of these patients, a subset completed a 35-item health questionnaire. Questionnaire data were available from 13,140 patients with the median length of time between cohort entry and questionnaire completion being 2.65 years. Patients missing responses to the questions on medication adherence $(n=981)$ were excluded, leaving 12,159 patients for analysis. This study was approved by the ethics committees at Kaiser Permanente Northern and Southern California (reference numbers CN-09AGo-14-H and 5572, respectively). A waiver of written informed consent was obtained due to the nature of the study being a minimal-risk health questionnaire.

\section{Medication adherence}

Medication adherence was assessed using three questions adapted from the Coronary Artery Risk Development in Young Adults (CARDIA) study [36]: (1) 'In the past month, how often did you take your medications as the doctor prescribed?' with the response options of 'All of the time (100\%),' 'Nearly all of the time (90\%),' 'Most of the time (75\%)', 'About half the time (50\%)' and 'Less than half the time $(<50 \%)$ '; (2) 'In the past month, how often did you forget to take one or more of your prescribed medications?' with the response options of 'Never', 'Once,' '2-3 times,' 'Once per week,' 'Several times per week' and 'Nearly every day'; and (3) 'In the past month, how often did you decide to skip one or more of your 
Kaiser Permanente Northern and Southern California patient

diagnosed with atrial fibrillation (AF) or atrial flutter between

January 1, 2006 and June 30, 2009: $\geq 1$ primary hospital discharge diagnosis(es) or $\geq 2$ outpatient diagnoses $(\mathrm{n}=99,365)$

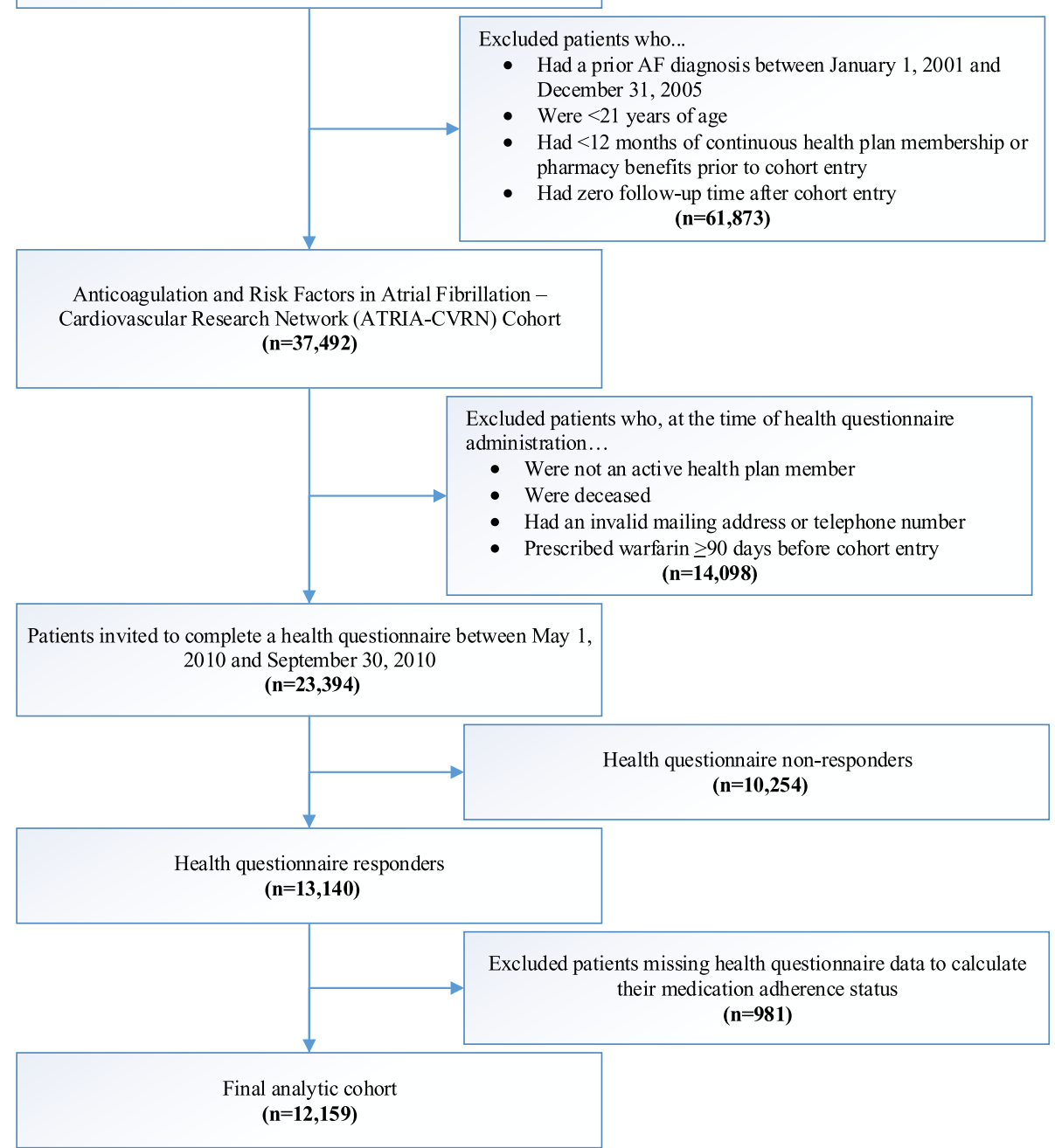

Fig. 1 Assembly of the adult atrial fibrillation cohort with complete health questionnaire data to assess medication adherence status ( $n=12,159)$

prescribed mediations' with the same response options as question two. Medication non-adherence was defined as either (a) response to question one of "most of the time (75\%)" or less, (b) response to question two of "once per week" or more, or (c) response to question three of "once per week" or more, based on previously validated definitions of non-adherence [37].

\section{Patient characteristics}

Age and sex were determined from the patient's electronic health record (EHR) at the time of AF diagnosis. Socio-demographic characteristics including race/ ethnicity, marital status, educational attainment, and household income were obtained by questionnaire. Physical activity (based on frequency, duration and intensity of activity in the past month) and height, weight, cigarette, alcohol and aspirin use during the year prior were also obtained by questionnaire. Height and weight were used to calculate body mass index $\left(\right.$ BMI $\left.\mathrm{kg} / \mathrm{m}^{2}\right)$. Self-reported health status was also collected from the questionnaire to evaluate frequency of poor physical and mental health in the past month prior to questionnaire completion (range: 0 to 30 days), overall current health (range: poor to excellent), if sleep was affecting daily function (range: never to almost every day), memory decline and health literacy (based on a validated 3-item instrument examining problems due to reading, understanding and completing medical forms dichotomized into adequate or inadequate [38-41]). 
History of coronary heart disease, chronic heart failure, dementia, depression, diabetes mellitus, hypertension, ischemic stroke and transient ischemic attack were extracted from the EHRs using ICD-9-CM codes obtained from both inpatient and outpatient encounters during the 5-year period prior to questionnaire completion. These data were used to calculate a $\mathrm{CHADS}_{2}$ stroke risk score [26]. A Charlson Comorbidity Index (CCI) score was also calculated using ICD-9-CM codes from both inpatient and outpatient encounters during the 1-year period prior to questionnaire completion, to account for overall comorbidity burden and risk of mortality [42].

\section{Statistical analysis}

Socio-demographic characteristics, health behaviors, self-reported health status and medical history were compared across medication adherence status using chi-square, Fisher's exact and Wilcoxon signed-rank tests, as appropriate. Adjusted odds ratios (ORs) and 95\% confidence intervals (CIs) for non-adherence were estimated using multivariable logistic regression models among all patients with complete questionnaire data, with all patient characteristics available in the survey included as candidate predictors. All analyses were conducted using SAS software version 9.2 (SAS Institute, Cary, NC).

\section{Results}

Of the 12,159 patients with incident diagnosed AF who responded to the questionnaire items regarding medication adherence, 771 (6.3\%) were categorized as not adherent to their prescribed medications (Table 1). Patients who self-reported non-adherence to prescribed medications were younger ( $<65$ years of age) and more likely to be a racial/ethnic minority, not married or living with a partner and have a household income of $\$ 25,000$ or less compared to those who were adherent (Table 2). Non-adherent patients were also more likely to report less physical activity, be a current smoker and consume alcohol. In addition, non-adherent patients were more likely to self-report more than 1 day of poor physical and/or mental health in the last month, have fair or poor current overall health, indicate that sleep affected their daily function more than 1 day a week, have memory decline in the past 2-3 years and have inadequate health literacy compared to those patients who were adherent. Lastly, non-adherent patients were less likely to have hypertension, but more likely to report low-dose aspirin use in the last year, have a BMI $\geq 30$, $\mathrm{CHADS}_{2}$ score of 0 , depression and diabetes mellitus compared to adherent patients. Most univariate associations were retained in the multivariable model, with a few exceptions (Table 3). In particular, household income, cigarette use, self-rated current health, BMI and
Table 1 Responses to medication adherence questions from 12,159 incident atrial fibrillation patients

\begin{tabular}{ll}
\hline & $\mathrm{n}(\%)$ \\
\hline Question & \\
1. In the past month, how often did you take your medications as the \\
doctor prescribed? & $10,120(83.2)$ \\
All of the time (100\%) & $1726(14.2)$ \\
Nearly all of the time (90\%) & $202(1.7)$ \\
Most of the time (75\%) & $37(0.3)$ \\
About half the time (50\%) & $74(0.6)$ \\
Less than half the time (<50\%) & \\
2. In the past month, how often did you forget to take one or more \\
prescribed medications? & $7147(58.8)$ \\
Never & $3061(25.2)$ \\
Once & $1551(12.8)$ \\
2-3 times & $281(2.3)$ \\
Once per week & $73(0.6)$ \\
Several times per week & $46(0.4)$ \\
Nearly every day & $108(0.9)$ \\
3. In the past month, how often did you decide to skip one or more \\
prescribed medications?
\end{tabular}

${ }^{a}$ Not adherent defined as (a) an answer to question 1 as "most of the time $(75 \%)$ " or less or (b) an answer to question 2 as "once per week" or more or (c) an answer to question 3 as "once per week" or more

depression status were not statistically significant independent correlates of medication non-adherence. However, having a CCI score $\geq 3$ was associated with a decreased adjusted odds of medication non-adherence, after accounting for other patient characteristics.

\section{Discussion}

Among a large, ethnically-diverse sample of adults with incident AF receiving medical care within an integrated healthcare delivery system, we classified $6.3 \%$ as being not optimally adherent to prescribed medications per self-report. Patients were more likely to be non-adherent to their prescribed medications if they were a racial/ethnic minority versus non-Hispanic white, not married/with partner, physical inactive, used alcohol, had any days of self-reported poor physical health, mental health and/or sleep quality in the past 30 days 
Table 2 Characteristics of 12,159 incident atrial fibrillation patients by medication adherence status

\begin{tabular}{|c|c|c|c|}
\hline & $\begin{array}{l}\text { Adherent } \\
n=11,388 \\
(93.7 \%)\end{array}$ & $\begin{array}{l}\text { Not Adherent } \\
n=771 \\
(6.3 \%)\end{array}$ & $\mathrm{P}^{+}$ \\
\hline \multicolumn{4}{|l|}{ Socio-demographics } \\
\hline Age, years & & & $<0.001$ \\
\hline Median & 72.7 & 70.1 & \\
\hline 25th -75 th $\%$ & $64.4-79.9$ & $59.5-79.1$ & \\
\hline Age group, years, n (\%) & & & $<0.001$ \\
\hline$<65$ & 3017 (26.5) & $293(38.0)$ & \\
\hline $65-74$ & 3549 (31.2) & $198(25.7)$ & \\
\hline $75-84$ & $3523(30.9)$ & $185(24.0)$ & \\
\hline$\geq 85$ & $1299(11.4)$ & $95(12.3)$ & \\
\hline Male, n (\%) & $6498(57.1)$ & $435(56.4)$ & 0.735 \\
\hline Race/Ethnicity, n (\%) & & & $<0.001$ \\
\hline Non-Hispanic White & 8505 (74.7) & $502(65.1)$ & \\
\hline Non-Hispanic Black & $572(5.0)$ & $81(10.5)$ & \\
\hline Non-Hispanic Asian/Pacific Islander & $775(6.8)$ & $64(8.3)$ & \\
\hline Hispanic & $889(7.8)$ & $75(9.7)$ & \\
\hline Other/Unknown & $647(5.7)$ & $49(6.4)$ & \\
\hline Marital Status, n (\%) & & & 0.006 \\
\hline Married/Partner & 7470 (65.6) & $463(60.1)$ & \\
\hline Not Married/Partner & $3788(33.3)$ & $300(38.9)$ & \\
\hline Unknown & $130(1.1)$ & $8(1.0)$ & \\
\hline Educational Attainment, n (\%) & & & 0.299 \\
\hline Less than High School & $929(8.2)$ & $72(9.3)$ & \\
\hline High School Graduate & $2110(18.5)$ & $145(18.8)$ & \\
\hline Some College & $4075(35.8)$ & $290(37.6)$ & \\
\hline Bachelor's Degree or Higher & 3999 (35.1) & $243(31.5)$ & \\
\hline Unknown & $275(2.4)$ & $21(2.7)$ & \\
\hline Household Income, n (\%) & & & 0.008 \\
\hline$\$ 25,000$ or less & $1694(14.9)$ & 150 (19.5) & \\
\hline$\$ 25,001-50,000$ & 2477 (21.8) & $168(21.8)$ & \\
\hline$\$ 50,001-80,000$ & $1967(17.3)$ & $122(15.8)$ & \\
\hline More than $\$ 80,000$ & $2501(22.0)$ & $169(21.9)$ & \\
\hline Unknown & $2749(24.1)$ & $162(21.0)$ & \\
\hline Questionnaire Language, n (\%) & & & 0.349 \\
\hline English & $11,077(97.3)$ & $752(97.5)$ & \\
\hline Spanish & $280(2.5)$ & $19(2.5)$ & \\
\hline Mandarin & $31(0.3)$ & $0(0.0)$ & \\
\hline Region, n (\%) & & & 0.941 \\
\hline Northern California & $5854(51.4)$ & $395(51.2)$ & \\
\hline Southern California & $5534(48.6)$ & $376(48.8)$ & \\
\hline \multicolumn{4}{|l|}{ Health Behaviors } \\
\hline Physical Activity (past month), n (\%) & & & $<0.001$ \\
\hline None & $854(7.5)$ & $86(11.2)$ & \\
\hline Low/Moderate & $7683(67.5)$ & $532(69.0)$ & \\
\hline
\end{tabular}


Table 2 Characteristics of 12,159 incident atrial fibrillation patients by medication adherence status (Continued)

\begin{tabular}{|c|c|c|c|}
\hline & $\begin{array}{l}\text { Adherent } \\
n=11,388 \\
(93.7 \%)\end{array}$ & $\begin{array}{l}\text { Not Adherent } \\
n=771 \\
(6.3 \%)\end{array}$ & $\mathrm{P}^{\dagger}$ \\
\hline High & $2802(24.6)$ & $149(19.3)$ & \\
\hline Unknown & $49(0.4)$ & $4(0.5)$ & \\
\hline Cigarette Use (past year), n (\%) & & & 0.002 \\
\hline Never & $4758(41.8)$ & $303(39.3)$ & \\
\hline Former & $5759(50.6)$ & $383(49.7)$ & \\
\hline Current & $624(5.5)$ & $67(8.7)$ & \\
\hline Unknown & $247(2.2)$ & $18(2.3)$ & \\
\hline Alcohol Use (past year), n (\%) & & & $<0.001$ \\
\hline Never & $2364(20.8)$ & $105(13.6)$ & \\
\hline Former & $2245(19.7)$ & $169(21.9)$ & \\
\hline Current & $6585(57.8)$ & $484(62.8)$ & \\
\hline Unknown & $194(1.7)$ & $13(1.7)$ & \\
\hline \multicolumn{4}{|l|}{ Self-Reported Health Status } \\
\hline Poor Physical Health (past month), n (\%) & & & $<0.001$ \\
\hline 0 days & $6313(55.4)$ & $320(41.5)$ & \\
\hline 1-7 days & $2156(18.9)$ & $196(25.4)$ & \\
\hline $8-21$ days & $816(7.2)$ & $78(10.1)$ & \\
\hline $22-28$ days & $80(0.7)$ & $7(0.9)$ & \\
\hline$\geq 29$ days & $821(7.2)$ & $74(9.6)$ & \\
\hline Unknown & $1202(10.6)$ & $96(12.5)$ & \\
\hline Poor Mental Health (past month), n (\%) & & & $<0.001$ \\
\hline 0 days & $7835(68.8)$ & $400(51.9)$ & \\
\hline $1-7$ days & $1401(12.3)$ & $134(17.4)$ & \\
\hline $8-21$ days & $593(5.2)$ & $66(8.6)$ & \\
\hline $22-28$ days & $66(0.6)$ & $6(0.8)$ & \\
\hline$\geq 29$ days & $422(3.7)$ & $50(6.5)$ & \\
\hline Unknown & $1071(9.4)$ & $115(14.9)$ & \\
\hline Self-Rated Current Health, n (\%) & & & $<0.001$ \\
\hline Poor & $641(5.6)$ & $59(7.7)$ & \\
\hline Fair & $2581(22.7)$ & $228(29.6)$ & \\
\hline Good & $4561(40.1)$ & $271(35.2)$ & \\
\hline Very Good & $2890(25.4)$ & $168(21.8)$ & \\
\hline Excellent & $625(5.5)$ & $36(4.7)$ & \\
\hline Unknown & $90(0.8)$ & $9(1.2)$ & \\
\hline Sleep Affecting Daily Function (average), n (\%) & & & $<0.001$ \\
\hline Never & $2747(24.1)$ & $145(18.8)$ & \\
\hline Rarely & $5283(46.4)$ & $294(38.1)$ & \\
\hline 1-3 days/week & $1685(14.8)$ & $154(20.0)$ & \\
\hline 4-6 days/week & $431(3.8)$ & $44(5.7)$ & \\
\hline Almost every day & $1060(9.3)$ & $126(16.3)$ & \\
\hline Unknown & $182(1.6)$ & $8(1.0)$ & \\
\hline Memory Decline (past 2-3 years), n (\%) & & & $<0.001$ \\
\hline No & $5582(49.0)$ & $294(38.1)$ & \\
\hline
\end{tabular}


Table 2 Characteristics of 12,159 incident atrial fibrillation patients by medication adherence status (Continued)

\begin{tabular}{|c|c|c|c|}
\hline & $\begin{array}{l}\text { Adherent } \\
n=11,388 \\
(93.7 \%)\end{array}$ & $\begin{array}{l}\text { Not Adherent } \\
n=771 \\
(6.3 \%)\end{array}$ & $\mathrm{P}^{\dagger}$ \\
\hline Yes & $4601(40.4)$ & $397(51.5)$ & \\
\hline Unknown & $1205(10.6)$ & $80(10.4)$ & \\
\hline Health Literacy, n (\%) & & & $<0.001$ \\
\hline Adequate & $8813(77.4)$ & $536(69.5)$ & \\
\hline Inadequate & $2575(22.6)$ & $235(30.5)$ & \\
\hline \multicolumn{4}{|l|}{ Medical History } \\
\hline Low-Dose Aspirin (past year; $\leq 100$ mg/tablet), n (\%) & & & 0.043 \\
\hline Did not use in the last $12 \mathrm{mo}$. & $6462(56.7)$ & $404(52.4)$ & \\
\hline Did use in the last $12 \mathrm{mo}$. & $4488(39.4)$ & $339(44.0)$ & \\
\hline Unknown & $438(3.9)$ & $28(3.6)$ & \\
\hline Aspirin or Aspirin-Product (past year; $\geq 325$ mg/tablet), n (\%) & & & 0.240 \\
\hline Did not use in the last $12 \mathrm{mo}$. & $8431(74.0)$ & $551(71.5)$ & \\
\hline Did use in the last $12 \mathrm{mo}$. & $2287(20.1)$ & $174(22.6)$ & \\
\hline Unknown & $670(5.9)$ & $46(6.0)$ & \\
\hline Body Mass Index (kg/m²), n (\%) & & & 0.003 \\
\hline$<25$ & $3211(28.2)$ & $191(24.8)$ & \\
\hline $25-30$ & $3915(34.4)$ & $254(32.9)$ & \\
\hline$\geq 30$ & $3492(30.7)$ & $284(36.8)$ & \\
\hline Unknown & $770(6.8)$ & $42(5.5)$ & \\
\hline Charlson Comorbidity Index (past year), n (\%) & & & 0.124 \\
\hline 0 & $3773(33.1)$ & $255(33.1)$ & \\
\hline 1 & $2346(20.6)$ & $175(22.7)$ & \\
\hline 2 & $1652(14.5)$ & $124(16.1)$ & \\
\hline$\geq 3$ & $3617(31.8)$ & $217(28.2)$ & \\
\hline $\mathrm{CHADS}_{2}$ Score (past five years), $\mathrm{n}(\%)$ & & & 0.049 \\
\hline 0 & $1849(16.2)$ & $155(20.1)$ & \\
\hline 1 & 3959 (34.8) & $254(32.9)$ & \\
\hline 2 & $3782(33.2)$ & $245(31.8)$ & \\
\hline$\geq 3$ & $1798(15.8)$ & $117(15.2)$ & \\
\hline Coronary Heart Disease, n (\%) & $1372(12.1)$ & $92(11.9)$ & 0.955 \\
\hline Chronic Heart Failure, n (\%) & $2457(21.6)$ & $167(21.7)$ & 0.964 \\
\hline Dementia, n (\%) & $288(2.5)$ & $19(2.5)$ & $>0.999$ \\
\hline Depression, n (\%) & $2309(20.3)$ & $191(24.8)$ & 0.004 \\
\hline Diabetes Mellitus, n (\%) & $3096(27.2)$ & $236(30.6)$ & 0.041 \\
\hline Hypertension, n (\%) & 8961 (78.7) & $568(73.7)$ & 0.001 \\
\hline Ischemic Stroke or Transient Ischemic Attack, n (\%) & $604(5.3)$ & $39(5.1)$ & 0.868 \\
\hline
\end{tabular}

${ }^{\dagger} p$-values based on chi-square, Fisher's exact and Wilcoxon signed-rank tests

versus 0 days, had memory decline, inadequate health literacy, low-dose aspirin use and/or diabetes mellitus. Whereas, patients who were of older age (65-84 years versus $<65$ years of age), had a Charlson Comorbidity Index score $\geq 3$ versus 0 and had hypertension were less likely to be non-adherent.
Several studies have estimated one-year non-adherence rates to oral anticoagulants among AF patients to be 3$28 \%$ based on the type of oral anticoagulant prescribed [11-13, 43]. Although our study assessed general medication adherence, which included assessment of both medication adherence to AF-specific treatment and any 
Table 3 Adjusted odds ratios (95\% confidence intervals) predicting medication non-adherence among 12,159 atrial fibrillation patients

\begin{tabular}{ll}
\hline $\begin{array}{l}\text { Atrial Fibrillation Patients } \\
(n=12,159)\end{array}$ \\
\hline Socio-demographics
\end{tabular}

Socio-demographics

Age group, years

$<65$

65-74

$75-84$

$\geq 85$

Gender

$$
\text { Male }
$$

Female

Race/Ethnicity

Non-Hispanic White

Non-Hispanic Black

Non-Hispanic Asian/Pacific Islander

Hispanic

Other/Unknown

Marital Status

Married/Partner
Not Married/Partner
Unknown
Educational Attainment
Less than High School
High School Graduate
Some College
Bachelor's Degree or Higher
Unknown

Household Income

$$
\begin{aligned}
& \$ 25,000 \text { or less } \\
& \$ 25,001-50,000 \\
& \$ 50,001-80,000 \\
& \text { More than } \$ 80,000 \\
& \text { Unknown }
\end{aligned}
$$

Health Behaviors

Physical Activity (past month)

$\begin{array}{ll}\text { None } & 1.57(1.16,2.13)^{\mathrm{b}} \\ \text { Low/Moderate } & 1.13(0.93,1.38) \\ \text { High } & \text { REF } \\ \text { Unknown } & 1.81(0.61,5.37) \\ \text { Cigarette Use (past year) } & \\ \text { Never } & \text { REF } \\ \text { Former } & 0.98(0.83,1.16) \\ \text { Current } & 1.24(0.92,1.67) \\ \text { Unknown } & 1.00(0.59,1.70)\end{array}$

Table 3 Adjusted odds ratios (95\% confidence intervals) predicting medication non-adherence among 12,159 atrial fibrillation patients (Continued)

\begin{tabular}{ll}
\hline & $\begin{array}{l}\text { Atrial Fibrillation Patients } \\
(\mathrm{n}=12,159)\end{array}$ \\
\hline Alcohol Use (past year) & \\
Never & REF \\
Former & $1.69(1.30,2.20)^{\mathrm{a}}$ \\
Current & $1.91(1.51,2.43)^{\mathrm{a}}$ \\
Unknown & $1.36(0.72,2.58)$
\end{tabular}

Self-Reported Health Status

Poor Physical Health (past month)

$\begin{array}{ll}0 \text { days } & \text { REF } \\ 1-7 \text { days } & 1.43(1.17,1.75)^{\mathrm{a}} \\ 8-21 \text { days } & 1.28(0.96,1.72) \\ 22-28 \text { days } & 1.08(0.47,2.46) \\ \geq 29 \text { days } & 1.17(0.84,1.61) \\ \text { Unknown } & 1.10(0.83,1.46)\end{array}$

Poor Mental Health (past month)

$\begin{array}{ll}0 \text { days } & \text { REF } \\ 1-7 \text { days } & 1.31\end{array}$

$1.31(1.05,1.63)^{\mathrm{c}}$

8-21 days

$1.35(0.99,1.83)$

22-28 days

$0.96(0.40,2.34)$

$\geq 29$ days

$1.44(1.00,2.06)^{c}$

Unknown

$1.78(1.38,2.31)^{\mathrm{a}}$

Self-Rated Current Health

Poor $\quad 0.80(0.49,1.32)$

Fair

$0.94(0.63,1.40)$

Good

$0.79(0.54,1.15)$

Very Good

$0.94(0.64,1.37)$

Excellent

REF

Unknown

$1.20(0.54,2.69)$

Sleep Affecting Daily Function (average)

Never REF

Rarely $\quad 1.01(0.82,1.25)$

$1-3$ days/week $\quad 1.35(1.05,1.73)^{c}$

4-6 days/week $\quad 1.46(1.01,2.11)^{c}$

Almost every day $\quad 1.56(1.19,2.04)^{\mathrm{b}}$

Unknown

$0.55(0.24,1.26)$

Memory Decline (past 2-3 years)

No

REF

Yes

$1.34(1.13,1.59)^{\mathrm{a}}$

Unknown

$1.16(0.89,1.51)$

Health Literacy

Adequate REF

Inadequate $\quad 1.32(1.09,1.60)^{\mathrm{b}}$ 
Table 3 Adjusted odds ratios (95\% confidence intervals) predicting medication non-adherence among 12,159 atrial fibrillation patients (Continued)

Atrial Fibrillation Patients
$(\mathrm{n}=12,159)$

Baby or Low-Dose Aspirin (past year; $\leq 100 \mathrm{mg} /$ tablet)

$\begin{array}{ll}\text { Did not use in the last } 12 \mathrm{mo} . & \text { REF } \\ \text { Did use in the last } 12 \mathrm{mo} . & 1.21(1.03,1.42)^{\mathrm{c}} \\ \text { Unknown } & 1.01(0.66,1.56)\end{array}$

Aspirin or Aspirin-Product (past year; $\geq 325 \mathrm{mg} / \mathrm{tablet}$ )

$\begin{array}{ll}\text { Did not use in the last } 12 \mathrm{mo} . & \text { REF } \\ \text { Did use in the last } 12 \mathrm{mo} . & 1.03(0.85,1.25) \\ \text { Unknown } & 0.90(0.64,1.26) \\ \text { Body Mass Index }\left(\mathrm{kg} / \mathrm{m}^{2}\right) & \\ <25 & \text { REF } \\ 25-30 & 1.07(0.87,1.31) \\ \geq 30 & 1.20(0.96,1.48) \\ \text { Unknown } & 0.87(0.61,1.24)\end{array}$

Charlson Comorbidity Index (past year)

$\begin{array}{ll}0 & \text { REF } \\ 1 & 1.06(0.86,1.31) \\ 2 & 1.08(0.84,1.38) \\ \geq 3 & 0.77(0.60,1.00)^{c}\end{array}$

Coronary Heart Disease

$\begin{array}{ll}\text { No } & \text { REF } \\ \text { Yes } & 1.01(0.79,1.28)\end{array}$

Chronic Heart Failure

$\begin{array}{ll}\text { No } & \text { REF } \\ \text { Yes } & 0.98(0.80,1.20) \\ \text { Dementia } & \\ \text { No } & \text { REF } \\ \text { Yes } & 0.76(0.46,1.24) \\ \text { Depression } & \\ \text { No } & \text { REF } \\ \text { Yes } & 0.96(0.80,1.16)\end{array}$

Diabetes Mellitus

$\begin{array}{ll}\text { No } & \text { REF } \\ \text { Yes } & 1.22(1.01,1.48)^{\mathrm{c}} \\ \text { Hypertension } & \\ \text { No } & \text { REF } \\ \text { Yes } & 0.72(0.60,0.87)^{\mathrm{a}}\end{array}$

Ischemic Stroke or Transient Ischemic Attack

\begin{tabular}{ll} 
No & REF \\
Yes & $0.96(0.68,1.35)$ \\
\hline $\mathrm{a}<0.001$ & \\
$\mathrm{~b}<0.01$ & \\
$\mathrm{c}^{<}<0.05$ &
\end{tabular}

medication used to treat comorbidities, our medication non-adherence rate within our full AF cohort (6.3\%) was on the lower end of what has been previously estimated in an AF population. More broadly, it has been shown that self-reported medication adherence to cardiovascular disease medications is less than 40\% [44] and in an elderly population with a range of chronic illnesses only $45 \%$ had good medication adherence [45]. Our finding of only $6.3 \%$ medication non-adherence may be due, in part, to our cohort receiving care within an integrated healthcare delivery system that emphasizes coordination of care across different providers and clinical setting through a single EHR, as opposed to the more fragmented care seen in other types of healthcare delivery systems [46, 47]. Additionally, our assessment of medication adherence was based on self-report. This may have introduced some recall, social desirability and/or interviewer bias, leading to an over-estimate of the patient's actual medication adherence [48]. Nonetheless, using this brief self-reported measure of medication adherence increased our ability to capture adherence information from a larger population (in our case 12,159 atrial fibrillation patients) due to the ease and cost-effectiveness of implementation in a clinical setting [49]. Additionally, self-reported medication adherence measures may also have the benefit of demonstrating high specificity in capturing people who are truly non-adherent $[48,49]$. This was particularly valuable in our analyses, where we aimed to better understand population-level patient factors that may be associated with medication non-adherence in patients with atrial fibrillation. Self-reported adherence measures have also been shown to correlate well with adherence captured by pill counts and other monitoring devices [50], as well as with pharmacy dispensing records [51].

Results from the univariate analyses showed that patients who self-reported non-adherence to prescribed medications generally reported worse health indicators, as well as, factors related to low socio-economic status (racial/ethnic minority, being unmarried, lower household income, physical inactivity, BMI $\geq 30$, alcohol and cigarette use, poor physical, mental and current health, decreased sleep quality, memory decline, inadequate health literacy and both depression and diabetes mellitus) which are similar to those factors that have been reported with non-adherence in other chronic conditions [52]. Many of these risk factors for medication non-adherence may also be preventable and/or modifiable. Physical inactivity and alcohol use, although most likely not directly related to medication adherence, can lead to poor physical health, mental health and sleep quality. These later three factors have all been associated with medication non-adherence across populations [53, 54]. Poor health literacy, on the other hand, may be both modifiable and preventable. It is also one of the most common problems associated with medication non-adherence 
[55]. Additionally, our study found that non-adherent patients were less likely to have hypertension, but more likely to report low-dose aspirin use in the last year and have $\mathrm{CHADS}_{2}$ score of 0 when compared to patients who were adherent. The finding that patients who had hypertension were more likely to be adherent to their medication(s) is most likely due to the success of the Kaiser Permanente Hypertension Control Program [56]. From 2001 to 2013, hypertension control within KPNC increased from 44 to $90 \%$ [57]. One aspect of this program encouraged single pill combination therapy - combining multiple drugs into one pill. This strategy improved adherence, lowered patient costs and improved blood pressure control. Due to the program's success, Kaiser Permanente Southern California also implemented these strategies into their region. Thus, many, if not all, patients included in our study were recipients of this program.

In our multivariable regression analysis, results paralleled those from the univariate associations. Of note, however, was the finding that higher CCI score $(\geq 3)$ was linked to lower adjusted odds of being non-adherent compared to a CCI score of 0 . These results may reflect the impact of increased contact with healthcare providers, which can improve medication adherence accountability $[46,47]$.

Considering these findings, we also acknowledge that the cross-sectional and observational nature of these data precluded us from assessing longitudinal trends in medication adherence among our patients with incident AF. Additionally, we were unable to assess polypharmacy for inclusion in our adjusted models. Thus, it may be that our low non-adherence rate was influenced by the relatively few prescriptions prescribed per patient. We were also unable to measure time since diagnosis to questionnaire completion. As adherence rates to AF-specific medications have been shown to decline with time, it may be that many of our patients completed the questionnaire soon after being diagnosed with atrial fibrillation. Thus, adherence for those patients could have been misleadingly high. However, a strength of our study was that we had access to a large, socio-demographically diverse population of validated incident diagnosed AF patients. This allowed for the detailed investigation into the relationships between a wide range patient factors and medication adherence status within a highly representative population of AF patients.

\section{Conclusions}

In an ethnically-diverse cohort of AF patients, we identified multiple possible risk factors for medication non-adherence. These include selected socio-demographic characteristics, lifestyle factors, self-reported poor physical health, mental health and/or sleep quality, having memory decline, inadequate health literacy, using low-dose aspirin, having diabetes mellitus and a higher comorbidity burden. This has broad implications for both patients and providers when managing care for patients with $\mathrm{AF}$, where maximizing the benefit from AF medication and treatment for any existing comorbidities involves understanding the patient factors associated with medication non-adherence. Additionally, by finding ways to intervene on those risk factors that may be preventable and/or modifiable may improve overall medication adherence and bring awareness to those patients at highest risk for non-adherence.

\section{Abbreviations \\ AF: Atrial fibrillation; ATRIA-CVRN: Anticoagulation and Risk Factors in Atrial Fibrillation - Cardiovascular Research Network; BMI: Body mass index; CARDIA: Coronary Artery Risk Development in Young Adults; CCl: Charlson Comorbidity Index; Cl: Confidence interval; DOACs: Direct-acting oral anticoagulants; ECG: Electrocardiogram; EHR: Electronic health record; ICD-9- CM: International Classification of Diseases, Ninth Revision, Clinical Modification; KPNC: Kaiser Permanente Northern California; KPSC: Kaiser Permanente Southern California; OR: Odds ratio}

\section{Acknowledgements}

We would like to thank Sole Cardoso (Department of Research \& Evaluation, Kaiser Permanente Southern California) for providing administrative assistance with the preparation of this manuscript.

\section{Funding}

Research grant support from the National Heart, Lung, and Blood Institute of the National Institutes of Health (NIH; RC2HL 101589) and the American Recovery and Reinvestment Act (ARRA) of 2009.

\section{Availability of data and materials}

The data that support the findings of this study are available from the corresponding author on reasonable request.

\section{Authors' contributions}

Study concept and design: MHB, DES, ASG, MCF, KR. Acquisition of data: MHB, ASG, NU, RXW, KR. Statistical analysis: SRR, MHB, NU, I-LAL. Interpretation of data: All authors. Drafting of the manuscript: SRR, MHB, DES, ASG, MCF, TNH, KR. Critical revision of the manuscript: All authors. Obtained funding: DES, ASG, MCF, KR. Administrative, technical, or material support: ASG, KR. Study supervision: MHB, DES, ASG, TNH, KR. All authors read and approved the final manuscript.

\section{Ethics approval and consent to participate}

This study was approved by the ethics committees at Kaiser Permanente Northern and Southern California (reference numbers CN-09AGo-14-H and 5572, respectively). A waiver of written informed consent was obtained due to the nature of the study being a minimal-risk health questionnaire.

\section{Consent for publication}

Not applicable.

\section{Competing interests}

Stephanie R. Reading: Current employment at Amgen Inc.

Mary Helen Black: Current employment at Ambry Genetics.

Daniel E. Singer: Partial support by the Eliot B. and Edith C. Shoolman Fund

of Massachusetts General Hospital, Boston, MA; Consultant/advisory board role with Boehringer Ingelheim, Bristol-Myers Squibb, CVS Health, Johnson and Johnson, Merck and Pfizer; Research funding from Bristol-Myers Squibb and Boehringer Ingelheim.

Alan S. Go: Received research funding from the National Heart, Lung and Blood Institute; the National Institute of Diabetes, Digestive and Kidney Diseases; the National Institute on Aging and iRhythm Technologies. Kristi Reynolds: Received research funding from iRhythm Technologies. Margaret C. Fang, Natalia Udaltsova, Teresa N. Harrison, Rong Wei, In-Lu Amy Liu: No disclosures. 


\section{Publisher's Note}

Springer Nature remains neutral with regard to jurisdictional claims in published maps and institutional affiliations.

\section{Author details \\ ${ }^{1}$ Department of Research and Evaluation, Kaiser Permanente Southern California, 100 S. Los Robles Ave., 2nd floor, Pasadena, CA 91101, USA. 2Department of General Internal Medicine, Massachusetts General Hospital, Boston, MA, USA. ${ }^{3}$ Division of Research, Kaiser Permanente Northern California, Oakland, CA, USA. ${ }^{4}$ Departments of Epidemiology, Biostatistics and Medicine, University of California San Francisco, San Francisco, CA, USA. ${ }^{5}$ Division of Hospital Medicine, University of California San Francisco, San Francisco, CA, USA.}

\section{Received: 11 October 2018 Accepted: 4 February 2019} Published online: 11 February 2019

\section{References}

1. Go AS, Hylek EM, Phillips KA, Chang YC, Henault LE, Selby JV, et al. Prevalence of diagnosed atrial fibrillation in adults: national implications for rhythm management and stroke prevention: the anticoagulation and risk factors in atrial fibrillation (ATRIA) study. J Am Med Assoc. 2001;285(18):2370-5.

2. Chugh SS, Havmoeller R, Narayanan K, Singh D, Rienstra M, Benjamin EJ, et al. Worldwide epidemiology of atrial fibrillation: a global burden of disease 2010 study. Circulation. 2014;129(8):837-47.

3. Colilla S, Crow A, Petkun W, Singer DE, Simon T, Liu X. Estimates of current and future incidence and prevalence of atrial fibrillation in the U.S. adult population. Am J Cardiol. 2013;112(8):1142-7.

4. Mozaffarian D, Benjamin EJ, Go AS, Arnett DK, Blaha MJ, Cushman M, et al. Executive summary: heart disease and stroke statistics-2016 update: a report from the American Heart Association. Circulation. 2016;133:447-54.

5. Steinberg BA, Piccini J. Anticoagulation in atrial fibrillation. BMJ. 2014;14(348):g2116.

6. Prystowsky EN, Padanilam BJ, Fogel RI. Treatment of atrial fibrillation. JAMA. 2015;314:278-88

7. Van Gelder I, Rienstra M, Crijns H, Olshansky B. Rate control in atrial fibrillation. Lancet. 2006:388:818-28.

8. Chao TF, Liu CJ, Tuan TC, Chen SJ, Wang KL, Lin YJ, et al. Rate-control treatment and mortality in atrial fibrillation. Circulation. 2015;132(17):1604-12.

9. Glazer NL, Dublin S, Smith NL, French B, Jackson LA, Hrachovec JB, et al. Newly detected atrial fibrillation and compliance with antithrombotic guidelines. Arch Intern Med. 2007;167(3):246-52.

10. Broderick JP, Bonomo JB, Kissela BM, Khoury JC, Moomaw CJ, Alwell K, et al. Withdrawal of antithrombotic agents and its impact on ischemic stroke occurrence. Stroke. 2011:42:2509-14.

11. Schulman S, Shortt B, Robinson M, Eikelboom JW. Adherence to anticoagulant treatment with dabigatran in a real-world setting. J Thromb Haemost. 2013;11(7):1295-9.

12. Shore S, Carey EP, Turakhia MP, Jackevicius CA, Cunningham F, Pilote L, et al. Adherence to dabigatran therapy and longitudinal patient outcomes: insights from the veterans health administration. Am Heart J. 2014;167(6):810-7

13. Gorst-Rasmussen A, Skjøth F, Larsen TB, Rasmussen LH, Lip GYH, Lane DA. Dabigatran adherence in atrial fibrillation patients during the first year after diagnosis: a nationwide cohort study. J Thromb Haemost. 2015;13(4):495-504.

14. Kimmel SE, Chen Z, Price M, Parker CS, Metlay JP, Christie JD, et al. The influence of patient adherence on anticoagulation control with warfarin: results from the international normalized ratio adherence and genetics (INRANGE) study. Arch Intern Med. 2007;167(3):229-35.

15. Abdou JK, Auyeung V, Patel JP, Arya R. Adherence to long-term anticoagulation treatment, what is known and what the future might hold. Br J Haematol. 2016;174:30-42.

16. Lamori JC, Mody SH, Patel AA, Schein JR, Gross HJ, Dacosta Dibonaventura $M$, et al. Burden of comorbidities among patients with atrial fibrillation. Ther Adv Cardiovasc Dis. 2013;7(2):53-62.

17. Elezi S, Qerkini G, Bujupi L, Shabani D, Bajraktari G. Management and comorbidities of atrial fibrillation in patients admitted in cardiology service in Kosovo-a single-center study. Anadolu Kardiyol Derg. 2010:10(1):36-40.

18. Carroll K, Majeed A. Comorbidity associated with atrial fibrillation: a general practice-based study. Br J Gen Pract. 2001;51(472):884-6, 889-91.
19. Kim EJ, Yin X, Fontes JD, Magnani JW, Lubitz SA, McManus DD, et al. Atrial fibrillation without comorbidities: prevalence, incidence and prognosis (from the Framingham heart study). Am Heart J. 2016;177:138-44.

20. Sanfeliz-Gimeno G, Rodriguez-Bernal C, Hurtado I, Baizauli-Perez C, Librero J, Peiro S. Adherence to oral anticoagulants in patients with atrial fibrillation-a population based retrospective cohort study linking health information systems in the Valencia region. Spain: a study protocol. BMJ Open. 2015: 5(10):e007613. https://doi.org/10.1136/bmjopen-2015-007613.

21. American Heart Association. What is Atrial Fibrillation (AFib or AF)? | American Heart Association [Internet]. 2016. Available from: http://www. heart.org/en/health-topics/atrial-fibrillation/what-is-atrial-fibrillation-afib-or-af

22. Platt AB, Localio AR, Brensinger CM, Cruess DG, Christie JD, Gross R, et al. Risk factors for nonadherence to warfarin: results from the IN-RANGE study. Pharmacoepidemiol Drug Saf. 2008;17(9):853-60.

23. Chen S-Y, Wu N, Gulseth M, LaMori J, Bookhart BK, Boulanger L, et al. Oneyear Adherence to warfarin treatment for venous thromboembolism in high-risk patients and its association with long-term risk of recurrent events. J Manag Care Pharm. 2013;19(4):291-301.

24. Gumbinger $C$, Holstein $T$, Stock $C$, Rizos T, Horstmann S, Veltkamp R. Reasons underlying non-adherence to and discontinuation of anticoagulation in secondary stroke prevention among patients with atrial fibrillation. Eur Neurol. 2015;73(3-4):184-91.

25. MacEdo AF, Bell J, McCarron C, Conroy R, Richardson J, Scowcroft A, et al. Determinants of oral anticoagulation control in new warfarin patients: analysis using data from clinical practice research datalink. Thromb Res. 2015:136(2):250-60.

26. Gage BF, Van Walraven C, Pearce L, Hart RG, Koudstaal PJ, Boode BSP, et al. Selecting patients with atrial fibrillation for anticoagulation: stroke risk stratification in patients taking aspirin. Circulation. 2004;110(16):2287-92.

27. Deitelzweig SB, Buysman E, Pinsky B, Lacey M, Jing Y, Wiederkehr D, et al. Warfarin use and stroke risk among patients with nonvalvular atrial fibrillation in a large managed care population. Clin Ther. 2013;35(8):1201-10.

28. Orensky IA, Holdford DA. Predictors of noncompliance with warfarin therapy in an outpatient anticoagulation clinic. Pharmacotherapy. 2005;25:1801-8.

29. Gordon N, Lin T. The Kaiser Permanente Northern California Adult Member Health Survey. Perm J. 2016:20(4):34-42. https://doi.org/10.7812/TPP/15-225. Epub 2016 Aug 19.

30. Koebnick C, Langer-Gould AM, Gould MK, Chao CR, lyer RL, Smith N, et al. Sociodemographic characteristics of members of a large, integrated health care system: comparison with US Census Bureau data. Perm J. 2012:16(3):37-41.

31. Derose SF, Contreras R, Coleman KJ, Koebnick C, Jacobsen SJ. Race and ethnicity data quality and imputation using U.S. census data in an integrated health system: the kaiser permanente Southern California experience. Med Care Res Rev. 2013;70(3):330-45.

32. Singer DE, Chang Y, Borowsky LH, Fang MC, Pomernacki NK, Udaltsova N, Reynolds K, Go AS. A new risk scheme to predict ischemic stroke and other thromboembolism in atrial fibrillation: the ATRIA study stroke risk score. J Am Heart Assoc. 2013;2(3):e000250. https://doi.org/10.1161/JAHA.113. 000250.

33. Go AS, Hylek EM, Borowsky LH, Phillips KA, Selby JV, Singer DE. Warfarin use among ambulatory patients with nonvalvular atrial fibrillation: the anticoagulation and risk factors in atrial fibrillation (ATRIA) study. Ann Intern Med. 1999;131(12):927-34.

34. Reading SR, Go AS, Fang MC, Singer DE, ILA L, Black MH, et al. Health literacy and awareness of atrial fibrillation. J Am Heart Assoc. 2017;6(4):e005128.

35. Ashburner JM, Go AS, Reynolds K, Chang Y, Fang MC, Fredman L, et al. Comparison of frequency and outcome of major gastrointestinal hemorrhage in patients with atrial fibrillation on versus not receiving warfarin therapy (from the ATRIA and ATRIA-CVRN cohorts). Am J Cardiol. 2015;115(1):40-6.

36. Cutter GR, Burke GL, Dyer AR, Friedman GD, Hilner JE, Hughes GH, et al. Cardiovascular risk factors in young adults. The CARDIA baseline monograph. Control Clin Trials. 1991;12:1):1-77.

37. Gehi A, Haas D, Pipkin S, Whooley MA. Depression and medication adherence in outpatients with coronary heart disease: findings from the heart and soul study. Arch Intern Med. 2005;165(21):2508-13.

38. Chew LD, Bradley KA, Boyko E. Brief questions to identify patients with inadequate health literacy. Fam Med. 2004;16(2):438-40.

39. Chew LD, Griffin JM, Partin MR, Noorbaloochi S, Grill JP, Snyder A, et al. Validation of screening questions for limited health literacy in a large VA outpatient population. J Gen Intern Med. 2008;23(5):561-6. 
40. Hoover DS, Vidrine Jl, Shete S, Spears CA, Cano MA, Correa-Fernandez V, et al. Health literacy, smoking, and health indicators in African American adults. J Health Commun. 2015;20:24-33.

41. Wallace LS, Rogers ES, Roskos SE, Holiday DB, Weiss BD. Brief report: screening items to identify patients with limited health literacy skills. J Gen Intern Med. 2006;21(8):874-7.

42. Deyo RA, Cherkin DC, Ciol MA. Adapting a clinical comorbidity index for use with ICD-9-CM administrative databases. J Clin Epidemiol. 1992:45(6):613-9.

43. Al-Khalili F, Lindström C, Benson L. Adherence to anticoagulant treatment with apixaban and rivaroxaban in a real-world setting. Clin Trials Regul Sci Cardiol. 2016;18:1-4

44. Baroletti S, Dell'Orfano H. Medication adherence in cardiovascular disease. Circulation. 2010;121:1455-8.

45. Shruthi R, Jyothi R, Pundarikaksha HP, Nagesh GN, Tushar TJ. A study of medication compliance in geriatric patients with chronic illnesses at a tertiary care hospital. J Clin Diagnostic Res. 2016;10(12):FC40-3.

46. Gordon K, Smith F, Dhillon S. Effective chronic disease management: patients' perspectives on medication-related problems. Patient Educ Couns. 2007;65(3):407-15.

47. Brown MT, Bussell JK. Medication adherence: WHO cares? Mayo Clin Proc. 2011;86:304-14.

48. Hawkshead J, Krousel-Wood MA. Techniques for measuring medication adherence in hypertensive patients in outpatient settings: advantages and limitations. Dis Manag Health Out. 2007;15:109-18.

49. Nunes V, Neilson J, O'Flynn N, Calvert N, Kuntze S, Smithson H, Benson J, Blair J, Bowser A, Clyne W, Crome P, Haddad P, Hemingway S, Horne R, Johnson S, Kelly S, Packam B, Patel M, Steel J. Medicines adherence: Involving patients in decisions about prescribed medicines and supporting adherence. Other. London: NICE: National Institute for Health and Clinical Excellence; 2009. p. 1-30. Available from: http://eprints.hud.ac.uk/id/eprint/ 14569/1/PatelNICE43042.pdf.

50. Monnette A, Zhang Y, Shao H, Shi L. Concordance of Adherence measurement using self-reported Adherence questionnaires and medication monitoring devices: an updated review. PharmacoEconomics. 2018;36:17-27.

51. Rickles NM, Svarstad BL. Relationships between multiple self-reported nonadherence measures and pharmacy records. Res Soc Adm Pharm. 2007;3(4):363-77.

52. Jin J, Edward Sklar G, Min Sen OV, Chuen Li S. Factors affecting therapeutic compliance: a review from the patient's perspective. Ther Clin Risk Manag. 2008; $4: 269-86$

53. Grant AB, Williams NJ, Pandi-Perumal SR. Nonadherence to antihypertensive medications in adults with high risk for obstructive sleep apnea. J Clin Hypertens. 2017;19:540-2.

54. Holt EW, Muntner P, Joyce CJ, Webber L, Krousel-Wood MA. Health-related quality of life and antihypertensive medication adherence among older adults. Age Ageing. 2010;39(4):481-7.

55. Praska JL, Kripalani S, Seright AL, Jacobson TA. Identifying and assisting lowliteracy patients with medication use: a survey of community pharmacies. Ann Pharmacother. 2005;39:1441-5.

56. Jaffe MG, Lee GA, Young JD, Sidney S, Go AS. Improved blood pressure control associated with a large-scale hypertension program. J Am Med Assoc. 2013;310(7):699-705.

57. Jaffe MG, Young JD. The Kaiser Permanente northern California story: improving hypertension control from 44 to $90 \%$ in 13 years (2000 to 2013). J Clin Hypertens. 2016;18:260-1.

Ready to submit your research? Choose BMC and benefit from:

- fast, convenient online submission

- thorough peer review by experienced researchers in your field

- rapid publication on acceptance

- support for research data, including large and complex data types

- gold Open Access which fosters wider collaboration and increased citations

- maximum visibility for your research: over $100 \mathrm{M}$ website views per year

At BMC, research is always in progress.

Learn more biomedcentral.com/submissions 